\title{
Immune Cells in the Tumor Microenvironment and Cancer Stem Cells: Interplay for Tumor Progression
}

\author{
Deshmukh SK* \\ Department of Oncologic Sciences, Mitchell Cancer Institute, University of South \\ Alabama, Mobile, AL 36604, USA
}

*Corresponding Author: Sachin Kumar Deshmukh, Department of Oncologic Sciences, Mitchell Cancer Institute, University of South Alabama, 1660 SpringHill

\section{Editorial}

Volume 2 Issue 2

Received Date: July 09, 2018

Published Date: July 18, 2018

DOI: $10.23880 /$ jes-16000109

Avenue, Mobile, AL 36604-1405, USA; Tel: 251-445-9872; E-mail: skdeshmukh@health.southalabama.edu

Keywords: T Cells; Macrophages; MDSC; Cancer Stem Cells; Tumor Microenvironment

\section{Editorial}

Cancer is one of the leading causes of deaths worldwide due to poor clinical outcome attribute to ineffective treatment and resistance to therapy. Cancer stem cells (CSCs) which have the ability to give rise to all the cell types present in particular cancer believed to responsible for therapy resistance [1,2]. Cancer therapy resistance induced by CSCs eventually leads to cancer growth, relapse and metastatic spread. Other than CSCs, tumor microenvironment (TME) which consist cancer cell, fibroblast and immune cells influence the therapeutic outcome of cancer. Cancer cell secreted cytokines and growth factors attract the immune cells in TME. These immune cells get corrupted in TME and instead of killing, start helping cancer cell. In such circumstances understanding of the interaction of TME immune cells and CSCs is crucial, considering the fact that they both determine the cancer treatment results and their cross talk may provide the boost for unchecked growth of cancer cell.

One of the strategies cancer cells opt for immune evasion is to evade the recruitment of CD8+ cytotoxic T cells and natural killer (NK) cells. Even if these cells reach to the tumor site, the cancer cells nurture the immunosuppressive TME and induce the apoptosis of T cells by activating the immune checkpoints. The CSCs are demonstrated to avoid $\mathrm{T}$ cells by reducing the expression of MHC class I and MHC class II surface proteins, required for $\mathrm{T}$ cell activation [3]. Similarly, glioblastoma CSCs express a low level of MHC class I surface protein to avoid the NK cells activity [4]. Head and neck cancer CSCs were shown to express the programmed death-ligand 1 (PDL1), which binds to the programmed death (PD-1) receptor on $\mathrm{T}$ cells to restrain its function [5]. Recent data also shed the light on the interaction between CSCs and Treg, a subpopulation of $\mathrm{T}$ cells which regulate or suppress the activities of other immune cells that ultimately facilitate tumor cell growth. Under hypoxia, Treg cell produced IL-17 reported to increase the expansion of CSCs in colorectal cancer [6]. Further, glioblastoma CSCs polarize the macrophages to tumorassociated macrophages (TAM) or M2 type after activation STAT3 followed by cytokine production [7].

The TAM phenotype is known to support the tumor growth and metastasis by inducing immunosuppressive TME. Moreover, TAMs are reported to promote the selfrenewal of breast CSCs [8]. TAMs secreted immunosuppressive growth factor, milk-fat globule EGF-8 (MFG-E8) suggested eliciting the drug resistance in CSCs by activating several signaling pathways [9]. In syngeneic mammary tumor models, CSCs demonstrated to recruit Myeloid-derived suppressor cells (MDSCs), known for their immunosuppressive activities by the production of granulocyte colony-stimulating factor (G-CSF) [10]. In return, the MDSCs triggered Notch signaling that 


\section{Journal of Embryology \& Stem Cell Research}

promoted CSCs properties. Further in pancreatic cancer, MDSCs suggested to promote the stemness by increasing the expression of CSCs genes [11].

The TME act as a shelter for tumor cells specially CSCs that regulate the immune cells present in the TME. Growing body of evidence suggests that CSCs and TME significantly participate in tumor development, progression and therapy resistance. Further, an interaction of CSCs with immune cells in TME provides additional support to the tumor cells for unrestricted growth. Targeting the CSCs with novel therapeutic interventions represent an attractive strategy for the clinical outcome for cancer. Moreover, understanding of CSCs biology and its interaction with TME components may help in pinpointing the therapeutic targets for the cancer treatment.

\section{References}

1. Dawood S, Austin L, Cristofanilli M (2014) Cancer stem cells: implications for cancer therapy. Oncology 28(12): 1101-1107.

2. Deshmukh SK (2017) Breast cancer stem cells: understanding and opportunities for therapeutics. Journal of Stem Cell and Regenerative Biology 3(2): 163-164.

3. Di Tomaso T, Mazzoleni S, Wang E, Sovena G, Clavenna D, et al. (2010) Immunobiological characterization of cancer stem cells isolated from glioblastoma patients. Clinical Cancer Research 16(3): 800-813.

4. Castriconi R, Daga A, Dondero A, Zona G, Poliani PL, et al. (2009) NK cells recognize and kill human glioblastoma cells with stem cell-like properties. Journal of Immunology 182(6): 3530-3539.
5. Lee $\mathrm{Y}$, Shin JH, Longmire $\mathrm{M}$, Wang $\mathrm{H}$, Kohrt HE, et al. (2016) Cd44+ cells in head and neck squamous cell carcinoma suppress t-cell-mediated immunity by selective constitutive and inducible expression of PDL1. Clinical Cancer Research 22(14): 3571-3581.

6. Shaobo $Y$, Binquan $W$, Chunying $G$, Benyan $W$, Changhao C, et al. (2011) Foxp3+IL-17+ T cells promote development of cancer-initiating cells in colorectal cancer. Journal of Leukocyte Biology 89(1): 85-91.

7. Wu A, Wei J, Kong LY, Wang Y, Priebe W, et al. (2010) Glioma cancer stem cells induce immunosuppressive macrophages/microglia. Neuro-Oncology 12(11): 1113-1125.

8. Lu H, Clauser KR, Tam WL, Fröse J, Ye X, et al. (2014) A breast cancer stem cell niche supported by juxtacrine signalling from monocytes and macrophages. Nature Cell Biology 16(11): 1105-1117.

9. Jinushi M, Sato M, Kanamoto A, Itoh A, Nagai S, et al. (2009) Milk fat globule epidermal growth factor-8 blockade triggers tumor destruction through coordinated cell-autonomous and immune-mediated mechanisms. Journal of Experimental Medicine 206(6): 1317-1326.

10. Welte T, Kim IS, Tian L, Gao X, Wang H, et al. (2016) Oncogenic mTOR signalling recruits myeloid-derived suppressor cells to promote tumour initiation. Nature Cell Biology 18(6): 632-644.

11. Panni RZ, Sanford DE, Belt BA, Mitchem JB, Worley LA, et al. (2014) Tumor-induced STAT3 activation in monocytic myeloid-derived suppressor cells enhances stemness and mesenchymal properties in human pancreatic cancer. Cancer Immunology, Immunotherapy 63(5): 513-528. 